\title{
Synergy of CT and MRI in detecting trajectories of lodged bullets in decedents and potential hazards concerning the heating and movement of bullets during MRI
}

\author{
Gascho, Dominic ; Tappero, Carlo ; Zoelch, Niklaus ; Deininger-Czermak, Eva ; Richter, Henning ; Thali, \\ Michael J ; Schaerli, Sarah
}

\begin{abstract}
The purpose of this study was to assess the value of magnetic resonance imaging (MRI) in addition to computed tomography $(\mathrm{CT})$ in gunshot wound cases with bullets or pellets lodged inside the head. In this context, the potential heating and movement of the lodged bullets were additionally investigated using animal models. Eleven forensic cases of penetrating gunshot wounds underwent CT and MRI. The data of each imaging modality were reviewed according to the following relevant characteristics: bony lesion at the entrance, intracranial bone fragments, intracranial metal fragments, gunshot residues, the wound channel and the severity of metal artifacts. Four-point Likert scales were used for the assessment. The heating of projectiles and their magnetic field interactions with the static magnetic field were assessed using animal models. MRI presented major advantages in cases with transversal trajectories and non-ferromagnetic bullets compared to CT. In general, MRI enabled a clear visualization of the wound channel and gunshot-related soft tissue injuries. An image fusion of CT and MRI datasets demonstrated the individual strengths of both modalities. Radio frequency (RF)-induced heating due to bullets lodged inside the brain tissue was invalidated. The likelihood of ferromagnetic projectile migration inside brain tissue is low. MRI of decedents with a bullet lodged inside their heads is viable and provides a valuable supplement to CT. The in situ, noninvasive depiction of the wound channel and gunshot-related soft tissue injuries on MRI can contribute to the knowledge of wound ballistics.
\end{abstract}

DOI: https://doi.org/10.1007/s12024-019-00199-y

Posted at the Zurich Open Repository and Archive, University of Zurich

ZORA URL: https://doi.org/10.5167/uzh-178401

Journal Article

Accepted Version

Originally published at:

Gascho, Dominic; Tappero, Carlo; Zoelch, Niklaus; Deininger-Czermak, Eva; Richter, Henning; Thali, Michael J; Schaerli, Sarah (2020). Synergy of CT and MRI in detecting trajectories of lodged bullets in decedents and potential hazards concerning the heating and movement of bullets during MRI. Forensic Science, Medicine, and Pathology, 16(1):20-31.

DOI: https://doi.org/10.1007/s12024-019-00199-y 


\title{
Synergy of CT and MRI in detecting trajectories of lodged bullets in decedents and potential hazards concerning the heating and movement of bullets during MRI
}

\author{
Dominic Gascho ${ }^{1,2} \cdot$ Carlo Tappero ${ }^{1,3} \cdot$ Niklaus Zoelch ${ }^{1,4} \cdot$ Eva Deininger-Czermak ${ }^{1,5} \cdot$ Henning Richter $^{6}$. \\ Michael J. Thali ${ }^{1} \cdot$ Sarah Schaerli ${ }^{1}$ \\ Accepted: 28 October 2019 \\ (C) Springer Science+Business Media, LLC, part of Springer Nature 2019
}

\begin{abstract}
The purpose of this study was to assess the value of magnetic resonance imaging (MRI) in addition to computed tomography (CT) in gunshot wound cases with bullets or pellets lodged inside the head. In this context, the potential heating and movement of the lodged bullets were additionally investigated using animal models. Eleven forensic cases of penetrating gunshot wounds underwent CT and MRI. The data of each imaging modality were reviewed according to the following relevant characteristics: bony lesion at the entrance, intracranial bone fragments, intracranial metal fragments, gunshot residues, the wound channel and the severity of metal artifacts. Four-point Likert scales were used for the assessment. The heating of projectiles and their magnetic field interactions with the static magnetic field were assessed using animal models. MRI presented major advantages in cases with transversal trajectories and non-ferromagnetic bullets compared to CT. In general, MRI enabled a clear visualization of the wound channel and gunshot-related soft tissue injuries. An image fusion of CT and MRI datasets demonstrated the individual strengths of both modalities. Radio frequency (RF)-induced heating due to bullets lodged inside the brain tissue was invalidated. The likelihood of ferromagnetic projectile migration inside brain tissue is low. MRI of decedents with a bullet lodged inside their heads is viable and provides a valuable supplement to CT. The in situ, noninvasive depiction of the wound channel and gunshotrelated soft tissue injuries on MRI can contribute to the knowledge of wound ballistics.
\end{abstract}

Keywords Magnetic resonance imaging $\cdot$ Gunshot wound $\cdot$ Radiologic wound ballistics $\cdot$ Bullet $\cdot$ Projectile $\cdot$ Forensic radiology

Dominic Gascho

dominic.gascho@irm.uzh.ch

1 Department of Forensic Medicine and Radiology, Institute of Forensic Medicine, University of Zurich, Zurich, Switzerland

2 Department of Forensic Medicine and Imaging, Zurich Institute of Forensic Medicine, University of Zurich, Winterthurerstrasse 190/52, CH-8057 Zurich, Switzerland

3 Department of Radiology, Hôpital Fribourgeois, Fribourg, Switzerland

4 Department of Psychiatry, Psychotherapy and Psychosomatics, Hospital of Psychiatry, University of Zurich, Zurich, Switzerland

5 Institute of Diagnostic and Interventional Radiology, University Hospital Zurich, Zurich, Switzerland

6 Diagnostic Imaging Research Unit (DIRU), Clinic for Diagnostic Imaging, Vetsuisse Faculty, University of Zurich,

Zurich, Switzerland

\section{Introduction}

Postmortem computed tomography (CT) has demonstrated a high diagnostic value in identifying osseous lesions in gunshot wounds to the head, allowing conclusions concerning the ballistic trajectory of a bullet [1-3].

The detection of the entrance and exit wound, blood along the wound channel, intracranial bone splinters and intracranial bone lesion indicates the ballistic trajectory of a bullet [2, 4]. In the case of gunshot wounds with projectiles lodged inside the head, metallic foreign bodies are highlighted and easily located on CT scans [2, 4]. However, a projectile also causes severe metal artifacts on CT scans [5], and thus, the identification of the intracranial wound channel can be severely hampered. Assuming that a straight line between the entrance wound and the bullet terminus mirrors the actual bullet path is imprecise since a bullet may change its trajectory after ricocheting off the inner table of the skull or may slide along the inner table of the skull [6]. A retained bullet may also migrate postmortem along 
the wound channel due to gravity or mechanical manipulation [7]. In the case of lodged projectiles, further imaging modalities may be considered to supplement CT to detect soft tissue lesions and identify the intracranial trajectory.

Postmortem magnetic resonance imaging (MRI) demonstrated advantages to postmortem CT with regard to the depiction of the morphology and the detection of soft tissue lesions or hemorrhages [8-11]. Previous studies on phantoms and animal models demonstrated that non-ferromagnetic projectiles present only minimal metal artifacts [12-14] and that metal artifacts caused by ferromagnetic projectiles can be reduced using special MRI sequences and parameter settings [15]. Nevertheless, postmortem MRI is hardly performed in cases of gunshot wounds [16, 17], and thus far, only a few reported cases concerning MRI of decedents with a bullet lodged inside the body can be found in the literature $[4,7$, $18,19]$. In addition to limited access to MRI scanners, reasons for restraint in performing MRI on decedents with a bullet lodged inside the body are assuredly concerns regarding heating due to the applied radio frequency (RF) and movement of ferromagnetic bullets due to interactions with the static magnetic field of an MRI unit.

Severe RF-induced temperature increases were observed in studies on cardiac pacemakers and guidewires [20, 21]. A temperature increase to $63{ }^{\circ} \mathrm{C}$ was measured at the electrode tip of a cardiac pacemaker [20] and an increase to $48^{\circ} \mathrm{C}$ was measured at the tip of a guidewire [21]. According to investigations on electric marks, focal tissue damage can occur within $25 \mathrm{~s}$ when the temperature reaches a mere $50{ }^{\circ} \mathrm{C}$, and the focal electric mark is largely a thermal burn injury [22]. To date, only two studies have investigated the RF-induced heating of bullets using gel-filled phantoms [12, 14]. Smith et al. [12] investigated RF-induced heating by placing the bullets in contact with the bulb of an alcohol thermometer within an unspecified block of gel and reported no relevant heating of the studied bullets. More than 20 years later, Dedini et al. [14] investigated the RF-induced heating of five different bullets and detected a maximal temperature increase of only $1.7^{\circ} \mathrm{C}$; the bullets were embedded in a homogeneous phantom of gelled saline according to standard test methods of the American Society for Testing and Materials (ASTM) (ASTM F2182-11a). However, these in vitro measurements using the ASTM phantom can differ from measurements in inhomogeneous tissue with differences in electrical conductivity and thermal properties [23]. Together with the local electric field generated by the RF transmitter and the density of the tissue, the electrical conductivity of the tissue determines the specific absorption rate (SAR), which characterizes the deposited power in the tissue. At the tips or edges of metallic objects, high electric fields may occur, which will lead to a high local SAR in the surrounding tissue and finally induce a temperature increase depending on the thermal properties of the tissue [24, 25]. Accordingly, assessing the RF-induced heating of bullets in brain tissue was deemed necessary for this study.
The discrepancy between phantoms and biological tissue concerning the movement of ferromagnetic bullets due to the magnetic pull of the MRI unit was also discussed [26]. Previous studies investigated magnetic field interactions of projectiles in air $[14,27]$, according to ASTM standard test methods (ASTM F2052), or in ballistic gelatin [12, 28, 29]. Without a doubt, a lodged projectile experiences more friction in biological tissue than in air, and ordnance gelatin was developed as a substitute for biological tissue in high-velocity ballistic experiments. Thus, gelatin is not necessarily an appropriate substitute for biological tissue in low-velocity experiments, as would be the case in the migration of bullets due to the magnetic pull of the MRI unit. Bolliger et al. [26] used removed calf brains and pig livers to assess the potential movement of steel-jacketed projectiles in biological tissue. A bullet was manually inserted into the removed organs, which were placed in a plastic bucket within tissue paper, and exposed to the magnetic field of a 3 Tesla MRI scanner. They observed a migration of the ferromagnetic bullets several times; however, removed organs are drained of blood and thus differ in their tissue density compared to in situ organs. Additionally, the material that surrounds the organ, i.e. the fixation of the organ, may affect the likelihood of bullet migration within the organ. In light of this, removed organs may provide different results concerning the movement of a projectile within organs inside the body. Thus, it was deemed necessary to assess the magnetic field interactions of ferromagnetic bullets lodged in brain tissue that was still in situ.

This study consisted of three research topics. First, the diagnostic value of MRI in addition to $\mathrm{CT}$ in selected forensic cases of fatal gunshot injuries with projectiles lodged in the head was assessed, and the benefit of CT combined with MRI was demonstrated. Second, in this context, RF-induced heating due to retained bullets was investigated in situ using a porcine model, and third, magnetic field interactions of ferromagnetic bullets with the static magnetic field were assessed in situ using sheep models as a substitute for humans.

\section{Methods and materials}

\section{Study population}

This study included forensic cases with fatal gunshot wounds to the head. All cases were transported to our institute for postmortem imaging as part of the forensic judicial investigations. First, postmortem CT was performed. Decedents with a projectile, shot pellet or bullet fragment lodged inside the head were considered appropriate for this study. In consultation with the responsible case manager and under consideration of the available time before autopsy, an additional MRI was performed in selected cases $(n=11)$. In nine cases, the estimated postmortem interval (PMI) was less than 2 days (mean 
PMI: $27 \mathrm{~h}$; range: 6-47 h). One case had an estimated PMI of 3-5 days, and one case was examined in an advanced stage of decomposition with an estimated PMI of 1-2 weeks. Projectiles, shot pellets and larger bullet fragments were finally removed and delivered to a ballistic expert of the forensic institute. Before delivery, each projectile, pellet or bullet fragment was assessed concerning ferromagnetism using a permanent magnet.

\section{Imaging protocols and radiological assessments of forensic cases}

The CT data were acquired on a 128-slice scanner using zflying focal spot technology (Somatom Definition Flash, Siemens Healthcare, Forchheim, Germany). The scan parameters were $120 \mathrm{kVp}$ and $750 \mathrm{mAs}$. The raw data were reconstructed in an adjusted field of view with a $0.6 \mathrm{~mm}$ slice thickness using a hard kernel (H60) and a soft kernel (H31). MRI was performed on a 3 Tesla scanner (Achieva 3.0 TX, Philips Healthcare, Best, the Netherlands). The MRI in-house protocol included a T1-weighted inversion recovery sequence (TR: $2000 \mathrm{~ms}$, TE: $20 \mathrm{~ms}$ ) mainly for anatomy, a T2-weighted turbo spin echo sequence (TR: $3000 \mathrm{~ms}$, TE: $80 \mathrm{~ms}$ ) mainly for lesions, and a T2*-weighted fast field echo sequence $(\mathrm{TR}=909.8, \mathrm{TE}=16.1)$ for blood. All these sequences were acquired in an axial orientation. Additionally, an isotropic T2weighted spin echo (TR: $2500 \mathrm{~ms}$, TE: $226 \mathrm{~ms}$ ) sequence was performed to align the image to the bullet path.

The CT data and MRI data of each case were assessed by a radiologist with expertise in postmortem imaging and forensic medicine. The radiological foci were bone lesions at the entrance wound, intracranial bone fragments, intracranial metal fragments, gunshot residues, and the wound channel. A 4-point Likert scale was used to rate the detectability of the aforementioned findings on CT (hard and soft kernel reconstructions) and on MRI (T1weighted, T2-weighted and T2*-weighted sequences). The detectability of findings was graded as follows: $0=$ not visible, $1=$ barely identifiable, $2=$ indicated, and $3=$ clearly identifiable. Additionally, the severity of metal artifacts was assessed. A 4point Likert scale was also used to rate the severity of artifacts on CT (extent of streaks) and on MRI (extent of signal loss) according to their impairment concerning radiological diagnosis. The ratings were performed with regard to soft tissue assessments; thus, only the reconstruction with the soft kernel was used for CT ratings. For MRI ratings, a mean ranking for all three weightings was carried out. The severity of artifacts was graded as follows: $\mathrm{I}=$ minimal or not apparent, $\mathrm{II}=$ moderate, $\mathrm{III}=$ considerable, and IV = severe.

Finally, an image registration and fusion software (Syngo.via, Siemens Healthcare, Forchheim, Germany) [30, 31] was used to automatically superimpose the CT and MRI datasets to demonstrate the findings of both modalities at once. The intracranial trajectories were defined based on the $\mathrm{CT}$ and MRI findings and compared with the autopsy results.

\section{Heating tests in animal models}

A ballistic expert from the forensic institute was consulted for selecting frequently encountered bullets of different sizes and shapes. All selected bullets had been discharged in firing practice. Seven different projectiles were examined concerning heating during MRI scanning:

1) $8 \times 57 \mathrm{JS}$ bullet (standard hunting ammunition) $(8 \times$ $57 \mathrm{~mm})$

2) .308 Winchester bullet $(7.82 \times 51 \mathrm{~mm})$

3) GP90 bullet $(5.56 \times 45 \mathrm{~mm})$

4) GP90LSP bullet $(5.56 \times 45 \mathrm{~mm})$

5) 7.65 Browning bullet $(7.65 \times 17 \mathrm{~mm})$

6) PP41 bullet $(9 \times 19 \mathrm{~mm})$

7) .223 Remington bullet $(5.66 \times 45 \mathrm{~mm})$

All projectiles had lead cores and steel jackets, but the .223 Remington bullet had a jacket made of tombac. A pig head that was severed postmortem was used as a substitute for human tissue. The intracranial temperature of the pig head was approximately $13{ }^{\circ} \mathrm{C}$, which was measured using a $100 \Omega$ resistance temperature detector (Delta OHM, 35030 Caselle di Selvazzano (PD), Italy). To avoid temperature changes due to the adjustment of the projectile in the surrounding tissue, each projectile was put in a bucket filled with water at approximately $13{ }^{\circ} \mathrm{C}$ (similar to the temperature of the pig head) for a few minutes before testing. Temperature measurements of each bullet were performed using a dedicated fiber optic thermometer (Luxtron 812, LumaSense Technologies, Santa Clara, CA, USA). The small fiber optic probe was fixed with a tape on the back of the bullet, ensuring that the front part remained free of tape. The bullet with the fixed probe was manually inserted into the cerebellum through the foramen magnum of the severed pig head. Over a scan period of approximately $25 \mathrm{~min}$, the temperature was continuously measured 30 times per minute. The scan protocol consisted of the following standard sequences with a SAR below the maximum values defined by the International Commission on NonIonizing Radiation Protection (ICNIRP) [32]: T1-weighted (whole body SAR: $0.218 \mathrm{~W} / \mathrm{kg}$ ), T2-weighted (whole body SAR: $0.218 \mathrm{~W} / \mathrm{kg}$ ) and T2*-weighted (whole body SAR: $0.002 \mathrm{~W} / \mathrm{kg}$ ). The body coil was used for transmission, and an XL torso coil was used to receive the signal. The recorded temperatures were processed graphically using dedicated open source software (RStudio, Version 1.0.153, $\mathrm{R}$ Core Team, R Foundation for Statistical Computing, Vienna, Austria). 


\section{Movement tests in animal models}

Two ferromagnetic, steel-jacketed bullets were selected for evaluations concerning potential movement due to the magnetic pull. A GP11 bullet $(7.5 \times 55 \mathrm{~mm})$, which is elongate, and a PP41 bullet $(9 \times 19 \mathrm{~mm})$ were placed into each of seven severed sheep heads with a PMI of less than $12 \mathrm{~h}$ and one sheep head with a PMI of $80 \mathrm{~h}$. The bullets were manually inserted through the foramen magnum into the cerebellum. During this process, the orientation of the projectile was random with respect to the z-axis of the scanner. A CT scan was performed before and after the sheep head with the bullet lodged inside it was exposed to the magnetic field of the 3 Tesla MRI scanner. Every time a sheep head was exposed to the magnetic force of the scanner, it was placed in the middle of the MRI table, as would be the case in an MRI examination of a human head. The table was moved three times in and out of the MRI gantry. Each sheep head was used for both types of bullet. The GP11 bullet was inserted and tested first using half of the sheep heads. Then, the same four sheep heads were used a second time for the PP41 bullet. For the remaining half of the sheep heads, the order of bullet types was reversed. Consequently, upon insertion of the second bullet, the brain tissue had been previously lacerated due to manual insertion of a GP11 bullet in half of the heads and due to that of a PP41 bullet in the other half of the heads. The tests were repeated approximately every $24 \mathrm{~h}$ using the same sheep heads. The heads were stored in a cooler at $7{ }^{\circ} \mathrm{C}\left(44.6^{\circ} \mathrm{F}\right)$ between the tests, and the bullets were removed after each test by pulling on a thin thread fixed at the base of each bullet. The CT data from the scan before and after the bullet was exposed to the MRI magnetic field were reconstructed in an extended CT scale [33] for a clear depiction of the bullet and its position and orientation. The data sets were superimposed [30, 31] using dedicated software (Syngo.via, Siemens Healthcare, Forchheim, Germany) to reveal a movement of the bullet. A movement was defined as rotation if the bullet only rotated along its longitudinal axis without any visible migration. In contrast, a movement was defined as migration if the bullet visibly changed its position with or without an additional rotation.

\section{Results}

\section{Radiological assessments of forensic cases}

The results of the radiological assessments are listed in Table 1. CT was superior in detecting bone lesions at the entrance wound, bone fragments, metal fragments and gunshot residues compared to MRI. In contrast, MRI was superior in detecting the wound channel and soft tissue injuries. The superimposed reconstructions clearly demonstrated the diagnostic strength of both imaging modalities (Fig. 1). Autopsy confirmed all radiologically defined trajectories.

In 9/11 cases, the wound channel was not visible or was barely identifiable on CT. In two cases (case nos. 7 and 11), the wound channel was not visible with either imaging modality. The wound channel could not be identified in case no. 7 since the bullet did not penetrate intracranial tissue and was stuck in the facial bones. Case no. 10 was penetrated by numerous shot pellets. Nevertheless, the soft tissue could be assessed on MRI, and the pellets caused only minimal artifacts compared to the severe artifacts observed on the CT scan (Fig. 2). In case no. 11, the tissue remains of the heavily decomposed brain did not allow any conclusion on the wound channel or gunshot-related soft tissue lesions (Fig. 3). With regard to artifacts, MRI presented only minimal ( $8 / 11$ cases) to moderate (2/11 cases) artifacts, but in case no. 3 , the artifacts were graded as severe (Fig. 4). This particular case was the only case in which the projectile was ferromagnetic (steeljacketed). Nevertheless, movement of the bullet was not detected, and no thermal injuries due to heating were revealed at autopsy. In comparison, the artifacts were graded considerable (3/11 cases) to severe (5/11 cases) on CT scans in the majority of cases. In particular, cases with transversal trajectories yielded severe artifacts on the CT scans, and the artifacts substantially impeded the radiological assessment of the relevant findings (Fig. 5).

\section{Heating test results}

The range between the minimum and maximum temperatures was $<1{ }^{\circ} \mathrm{C}$ for all bullets $\left(8 \times 57 \mathrm{JS}\right.$ bullet: $14.7-15.3^{\circ} \mathrm{C}$; .308 Winchester bullet: $15.1-15.9{ }^{\circ} \mathrm{C}$; GP90 bullet: $12.2-12.6{ }^{\circ} \mathrm{C}$; GP90LSP bullet: $11.8-12.2^{\circ} \mathrm{C}$; 7.65 Browning bullet: $13.4-$ $13.9^{\circ} \mathrm{C}$; PP41 bullet: $13.1-13.5^{\circ} \mathrm{C}$; and .223 Remington bullet: $10.9-11.3^{\circ} \mathrm{C}$ ) (Fig. 6). The initial temperatures varied slightly $\left(11.1-15.84^{\circ} \mathrm{C}\right)$ between the bullets, possibly depending on the location and time in the water-filled bucket.

\section{Movement test results}

Overall, 96 movement tests were carried out (Table 2). Bullets rotated or aligned to the $\mathrm{z}$-axis of the scanner on 72 occasions (GP11 bullet: 38 times; PP41 bullet: 34 times), and migration was observed on 18 occasions (both bullets: 9 occasions). On five occasions, the bullet migrated through the entire brain (approximately $9 \mathrm{~cm}$ ), whereas in some other tests, the migration distance was less than $3 \mathrm{~cm}$. In five tests, the $P P 41$ bullet did not rotate or migrate after exposure to the magnetic force of the MRI scanner; in one test, the same outcome was observed for the GP11 bullet (Fig. 7). During all the tests on the sheep heads with a PMI of $<12 \mathrm{~h}$, the PP41 bullet migrated only once. Similarly, the GP11 bullet migrated once in a sheep head with a PMI of $<12 \mathrm{~h}$, although in the same head, the 


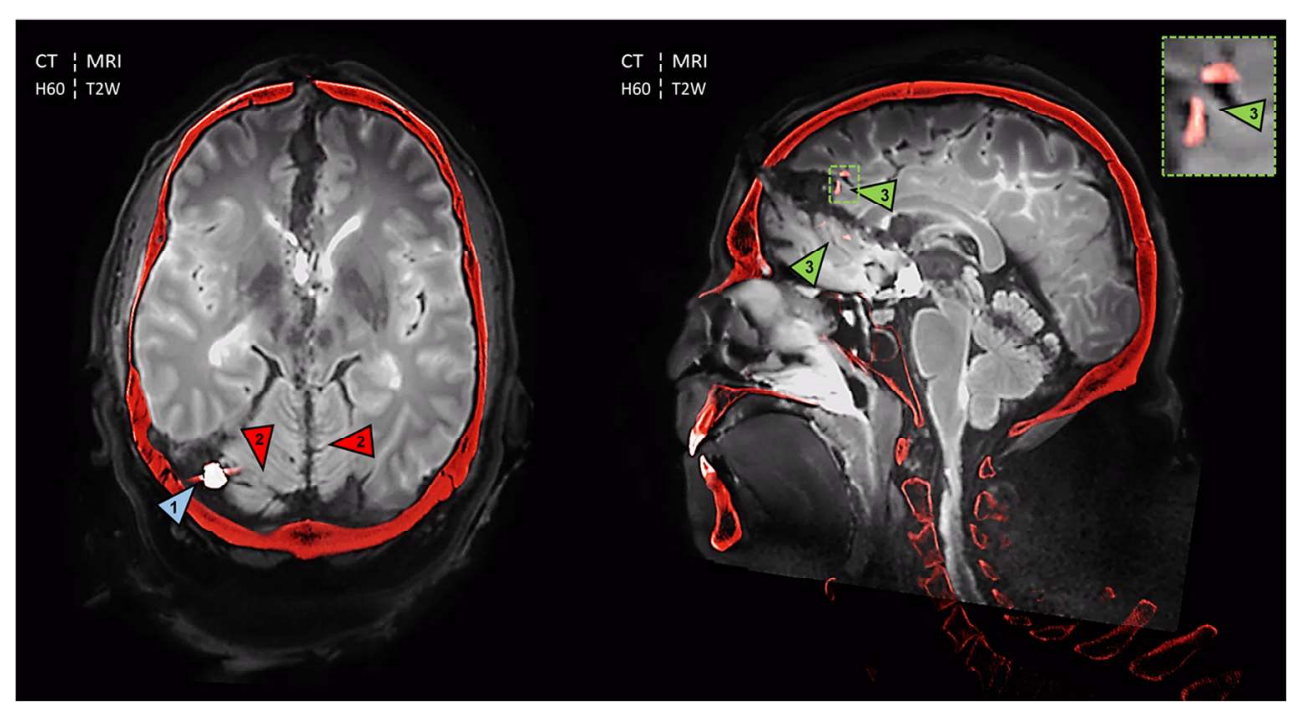

Fig. 1 The fusion of CT and MRI (CT bone (H60): highlighted in red; MRI: 3D T2-weighted (T2W)) aligned to the bullet path demonstrates the diagnostic strength of both imaging modalities in cases of penetrating gunshot wounds. By windowing into a higher range, the soft tissue visually disappeared on CT, while osseous lesions, bone fragments and the projectile, pellets or bullet fragments were still clearly visible. The now invisible soft tissue from CT was replaced by the detailed soft tissue from MRI by automated image fusion. While postmortem CT depicts the inwardly beveled cranial bone fracture at the entrance, locates the lodged

\section{Discussion}

PP41 bullet, which had been tested first, did not migrate. Up to a PMI of several days, neither the PP41 bullet nor the GP11 bullet migrated in four of the eight heads.

This article demonstrates that postmortem MRI is a valuable supplement to postmortem CT for gunshot wounds with bullet (no. 1: blue arrowhead) and highlights bone splintering (no. 3: green arrowheads) inside the soft tissue of the brain, postmortem MRI clearly illustrates the wound channel and the gunshot-related soft tissue injuries of the brain in this case of an intracranial ricochet (case no. 8). Due to severe metal artifacts on postmortem CT, the soft tissue injuries of the cerebellum were not visible; thus, the wound channel through the cerebellum was not visible on CT but was visible on MRI (no. 2: red arrowheads)

transversal trajectories and non-ferromagnetic projectiles that are lodged inside the body. The RF-induced heating of bullets in brain tissue when the bullet is centered in the bore of the scanner and near the isocenter during the examination, as would be the case in a standard MRI examination of the head, was invalidated. A migration of a ferromagnetic bullet inside the brain tissue due to the magnetic pull of the scanner is rather unlikely at a short PMI, while an alignment to the z-axis of the scanner is quite likely, especially for longish projectiles.



Fig. 2 Case no. 10 demonstrates severe bone and soft tissue destruction from numerous shotgun pellets (transversal view; left: CT bone (H60); middle: CT soft tissue (H31); right: MRI T2-weighted (T2W)). While CT allows the depiction of the osseous lesions, the soft tissue is not assessable due to severe metal artifacts caused by the pellets. In contrast, MRI allows visualization of the soft tissue, as the metallic shot pellets cause only moderate susceptibility artifacts. Although it is not possible to visualize each individual wound channel due to the large number of pellets, the soft tissue destruction still allows the analysis of the impact of the gunshot 


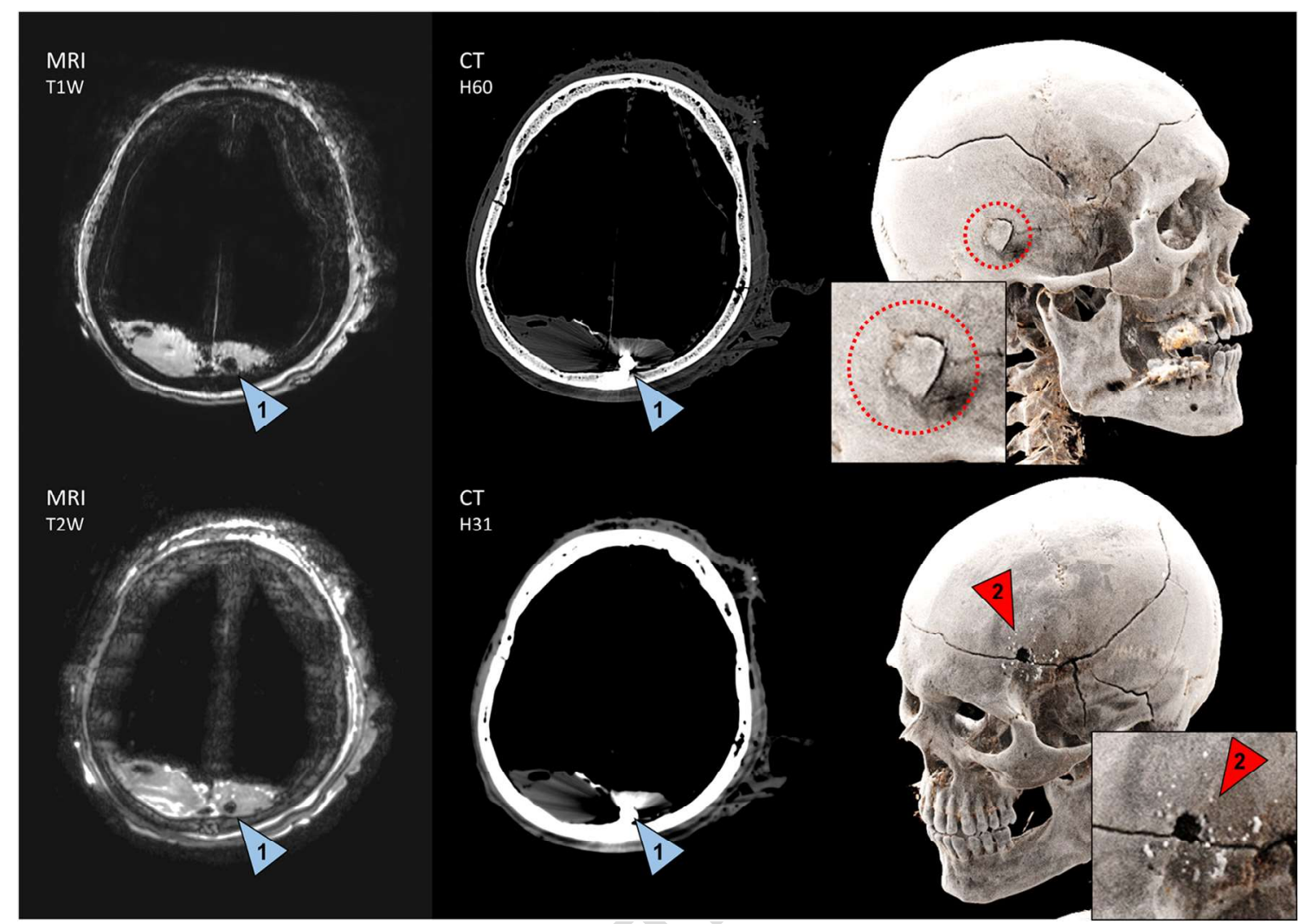

Fig. 3 Case no. 11 underwent imaging in an advanced state of decomposition (left column: MRI T1-weighted (T1W) and T2-weighted (T2W); middle column: CT bone (H60) and soft tissue (H31); right column: CT cinematic renderings). The retained bullet is visible on CT and MRI (no.1: blue arrowheads). At this stage of decomposition, the soft

The present study suggests soft tissue visualization with MRI as a replacement for soft tissue visualization with $\mathrm{CT}$ in fused image reconstructions. The combination of bone and metal visualizations on $\mathrm{CT}$ and the soft tissue depiction on MRI in a fused reconstruction demonstrates the diagnostic advantages of both imagining modalities. The main disadvantage of CT in cases with transversal trajectories compared to caudocranial trajectories can be explained by the fact that a retained bullet causes streaking artifacts along the slice orientation, and thus the transversal orientation, that extend over the whole CT image and impede the identification of relevant findings [34]. Although special iterative metal artifact reconstruction algorithms may reduce the extent of these streaks on CT [34], MRI is still superior concerning the visualization of the wound channel and soft tissue injuries. While nonferromagnetic bullets present fewer artifacts on MRI compared to CT, ferromagnetic bullets also cause severe artifacts on MRI. However, these artifacts do not extend over the whole image slice as metal artifacts on CT do; thus, a diagnostic assessment of soft tissue injury beyond the signal loss caused by the ferromagnetic bullet is feasible (Fig. 3). Additionally, special metal artifact reduction techniques may reduce the extent of the signal loss in the case of ferromagnetic tissue of the brain was no longer diagnostically assessable. The 3D CT reconstructions (right column) impressively demonstrate the radiating fracture lines, the point of ricochet (red circles) and gunshot residues at the entrance (no. 2: red arrowheads)

projectiles [15]; hence, MRI may also be of value in the case of ferromagnetic projectiles. In this study, only one of the 11 cases involved a ferromagnetic steel-containing bullet, which indicates that non-ferromagnetic bullets are more frequently encountered in our region, albeit only a small number of cases was presented here.

The visualization and assessment of soft tissue injuries in real decedents can provide important knowledge for the field of wound ballistics. A recent study demonstrated [35] that common synthetic models ("surrogates") showed similarities in terms of perforation and penetration capacities but also revealed non-negligible differences concerning the deformation of bullets compared to real bodies. While synthetic models caused only slight deformation, real bodies presented a stronger deformation for the same type of bullet [34]. This is an important finding since the degree of deformation affects the velocity and energy of the bullet and thus has an influence on the characteristics of the injury [35].

The RF-induced heating of bullets of different sizes and shapes inside the brain tissue was invalidated only under the explicit conditions selected for this study. Interactions between metallic objects and the RF field inside the bore of a scanner during image acquisition vary according to several conditions. 


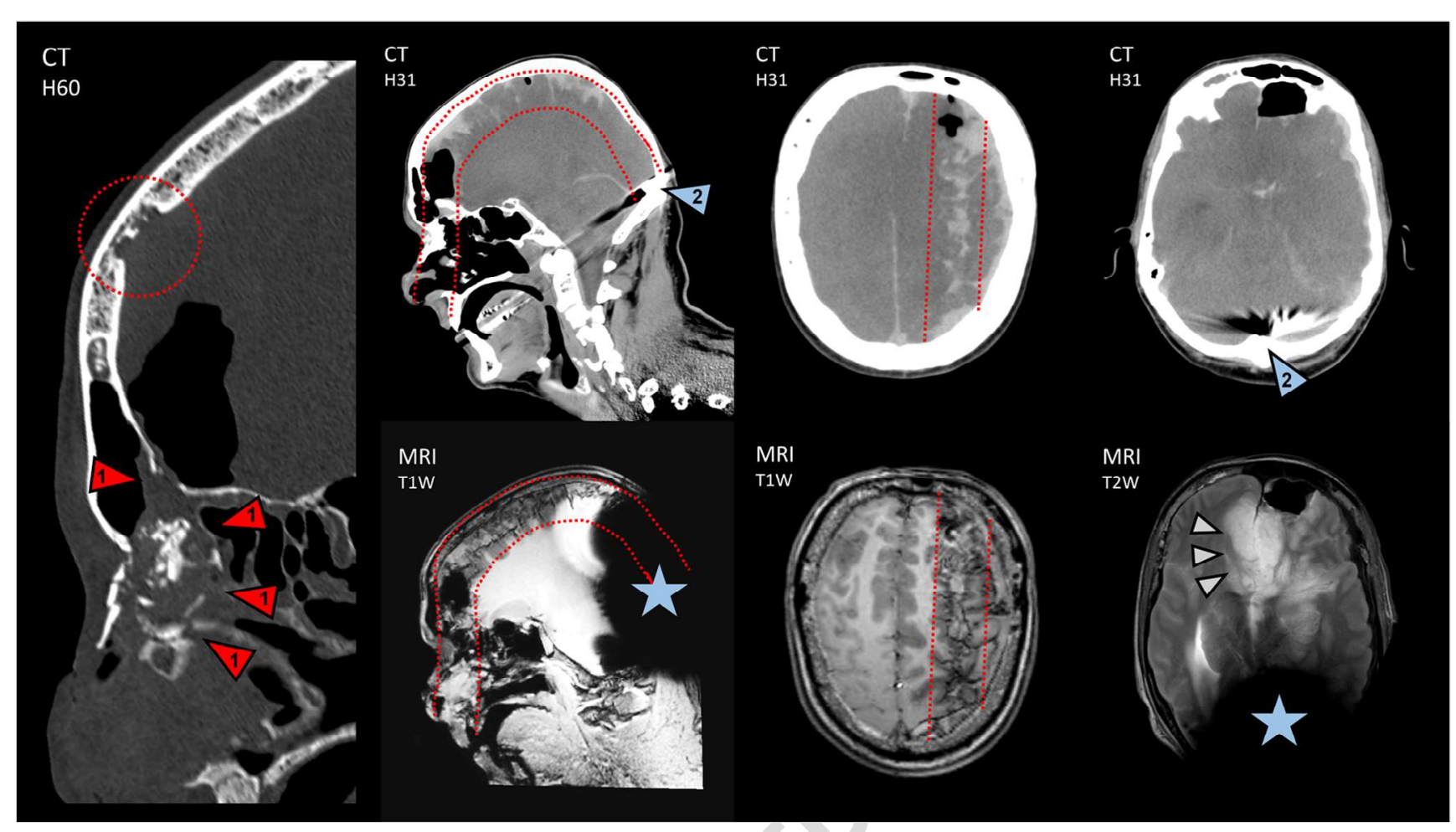

Fig. 4 Case no. 3 shows a gunshot through the left nostril (left image: CT bone (H60); upper row: CT soft tissue (H31); lower row: MRI T1weighted (T1W) and T2-weighted (T2W)). The point of ricochet is visible on the CT scan (red circle) as well as the osseous lesions and intracerebral gas at the bullet's entrance into the skull (no. 1: red arrowheads). Although the ferromagnetic bullet (no. 2: blue arrowhead) presents severe susceptibility artifacts on MRI (blue asterisks), the soft tissue injuries along the bullet path (red lines) at the frontal part of the brain were still visible on MRI. Blood was visible along the bullet path on CT, which allowed identification of the wound channel on both CT and MRI. However, MRI was superior for visualizing the other soft tissue lesions; for example, T2-weighted MRI presents an edema adjacent to the bullet path (small gray arrowheads)

With regard to the movement of ferromagnetic bullets inside an MRI unit, Bolliger et al. [26] observed that longish GP11 bullets as well as 9-mm Luger bullets, which have the same caliber as the PP41 bullets in the present study, exited removed calf brains almost every time they were positioned in a horizontal longitudinal orientation. In contrast, according to the results of this study, the migration of a ferromagnetic projectile in situ, and thus the creation of an artificial trajectory, is rather unlikely. Although the brain tissue was increasingly lacerated over the successive use of the sheep heads, migrations occurred in few tests. These results indicate that removed brains are not appropriate for studying the migration of projectiles in situ. Nonetheless, migration could not be completely excluded in situ, as migration depends on the degree of decomposition, the extent of tissue destruction, the size of the wound channel, the position of the bullet inside the head and the course of the trajectory. The strength of the magnetic pull varies according to the location of the bullet within the body and the position of the body within the MRI bore. The attraction is stronger at the outer range of the bore [26]; for example, a bullet lodged in the lateral part of the liver will experience more attraction than a bullet lodged in the brain. This should also be considered in cases of multiple gunshot wounds with more than one bullet lodged inside the body. 
Forensic Sci Med Pathol

Fig. 5 Especially in cases of horizontal trajectories (upper row: case no. 1; lower row case no. 6 ; left column: CT soft tissue (H31); right column: MRI T2-weighted (T2W)), the metal artifacts of the bullets (blue arrowheads) on CT (left column) severely impede the assessment of the bullet path (red lines). On MRI (right column), the susceptibility artifacts of the non-ferromagnetic bullets (blue asterisks) are only minimal to moderate, which enables visualization of the wound channel and clearly shows soft tissue injuries along the bullet's trajectory



Since only a migration will cause an artificial trajectory, which may lead to a false diagnostic assessment, the rotation of a bullet, which was frequently observed, was considered much less relevant. Performing a postmortem CT before a postmortem MRI examination to document the positions of lodged bullets or bullet fragments is recommended.
454 455 456
Fig. 6 The temperature of the bullet was documented 30 times per minute over a period of $25 \mathrm{~min}$. None of the bullets exhibited a relevant change in temperature during the MRI examination using standard sequences. It appears that the .308 Winchester bullet and the $8 \times 57$ $J S$ bullet demonstrated a minimal decrease in temperature, which probably occurred due to the lower temperature of the brain tissue $\left(13^{\circ} \mathrm{C}\right)$, since the temperature of the bullet may adjust to the temperature of the tissue. All bullets presented $13 \pm$ $2.5^{\circ}$ in accordance with the temperature of the pig head $\left(13^{\circ}\right)$ after 25 min of scanning

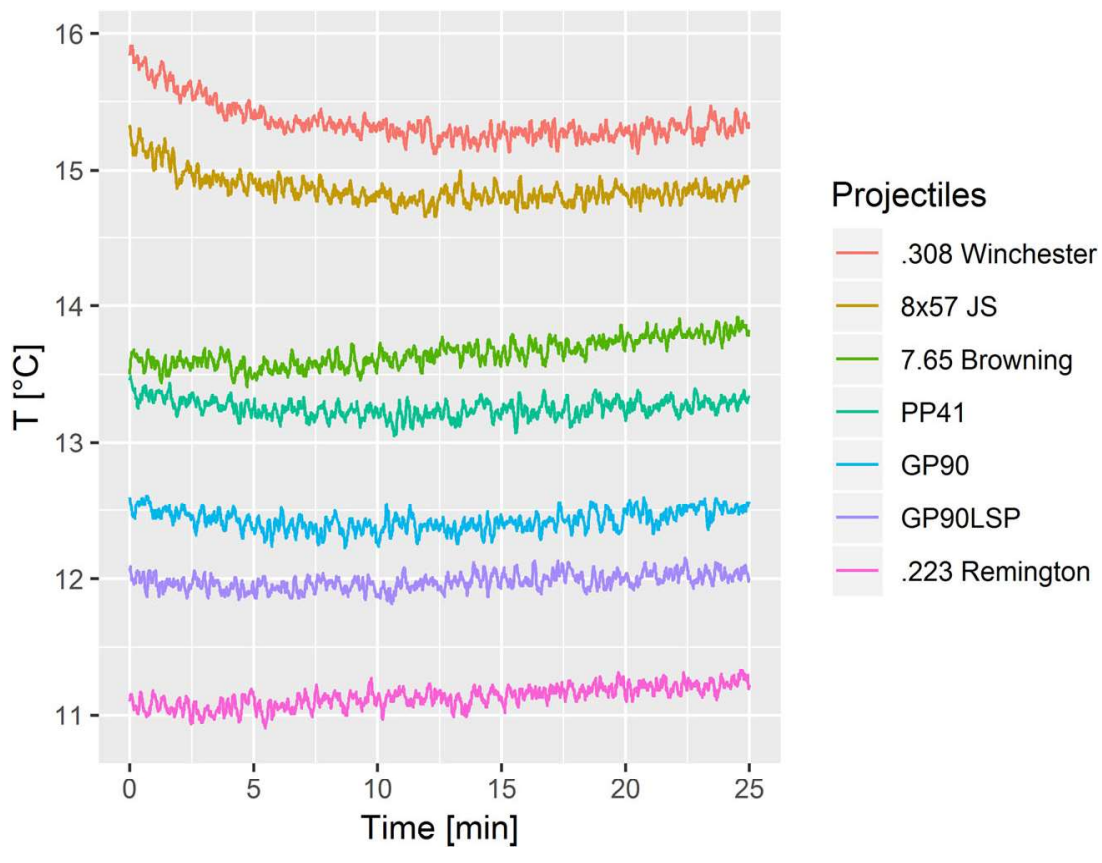


Forensic Sci Med Pathol

Fig. 7 Superimposed CT datasets of the scan before and after MRI (A-D). The CT data acquired before exposure to the MRI unit were inverted; thus, the initial position of the bullet is illustrated in black. In most cases, the bullets presented an alignment to the $\mathrm{z}$ axis of the scanner (A: GP11 bullet). Migrations were observed in only four of the eight heads (B: GP11 bullet; C: PP41 bullet). On one occasion and five occasions, the GP11 bullet and PP41 bullet, respectively, presented no change in position (D)

\begin{tabular}{|c|c|c|c|c|c|c|c|c|c|c|}
\hline Bullet & Test & PMI & $\begin{array}{l}\text { Head } \\
1^{*}\end{array}$ & $\begin{array}{l}\text { Head } \\
2^{*}\end{array}$ & $\begin{array}{l}\text { Head } \\
3^{*}\end{array}$ & $\begin{array}{l}\text { Head } \\
4^{*}\end{array}$ & $\begin{array}{l}\text { Head } \\
5 * *\end{array}$ & $\begin{array}{l}\text { Head } \\
6^{* *}\end{array}$ & $\begin{array}{l}\text { Head } \\
7 * *\end{array}$ & $\begin{array}{l}\text { Head } \\
8^{* *}\end{array}$ \\
\hline \multirow[t]{8}{*}{ GP11 } & 1 & $<12 \mathrm{~h}$ & $\circ$ & $\circ$ & - & n.t. & $\circ$ & $\circ$ & • & $\circ$ \\
\hline & 2 & $<36 \mathrm{~h}$ & $\circ$ & $\circ$ & n.t. & n.t. & $\circ$ & $\circ$ & • & n.t. \\
\hline & 3 & $<60 \mathrm{~h}$ & $\circ$ & • & $\circ$ & n.t. & $\circ$ & $\circ$ & n.t. & n.t. \\
\hline & 4 & $<84 \mathrm{~h}$ & $\circ$ & • & $\circ$ & $\circ$ & $\circ$ & - & n.t. & n.t. \\
\hline & 5 & $<108 \mathrm{~h}$ & $\circ$ & - & $\circ$ & n.t. & $\circ$ & $\circ$ & n.t. & $\circ$ \\
\hline & 6 & $<132 \mathrm{~h}$ & $\circ$ & • & n.t. & $\circ$ & $\circ$ & - & $\circ$ & • \\
\hline & 7 & $<156 \mathrm{~h}$ & n.t. & n.t. & n.t. & $\circ$ & n.t. & n.t. & n.t. & n.t. \\
\hline & 8 & $<180 \mathrm{~h}$ & n.t. & n.t. & $\circ$ & n.t. & n.t. & n.t. & n.t. & n.t. \\
\hline \multirow[t]{8}{*}{ PP41 } & 1 & $<12 \mathrm{~h}$ & $\circ$ & $\circ$ & $\circ$ & n.t. & $\circ$ & - & $\circ$ & $\circ$ \\
\hline & 2 & $<36 \mathrm{~h}$ & $\circ$ & - & n.t. & n.t. & $\circ$ & $\circ$ & $\circ$ & n.t. \\
\hline & 3 & $<60 \mathrm{~h}$ & - & • & $\circ$ & n.t. & . & $\circ$ & n.t. & n.t. \\
\hline & 4 & $<84 \mathrm{~h}$ & $\circ$ & - & $\circ$ & $\circ$ & 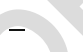 & • & n.t. & n.t. \\
\hline & 5 & $<108 \mathrm{~h}$ & $\circ$ & - & $\circ$ & n.t. & - & $\circ$ & n.t. & • \\
\hline & 6 & $<132 \mathrm{~h}$ & $\circ$ & - & n.t. & $\circ$ & - & $\circ$ & - & - \\
\hline & 7 & $<156 \mathrm{~h}$ & n.t. & n.t. & n.t. & 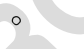 & n.t. & n.t. & n.t. & n.t. \\
\hline & 8 & $<180 \mathrm{~h}$ & n.t. & n.t. & $\circ$ & n.t. & n.t. & n.t. & n.t. & n.t. \\
\hline
\end{tabular}

$*=$ GP11 bullet was tested first, $* *=P P 41$ bullet was tested first, ${ }^{\circ}=$ rotation, $\cdot=$ migration, $-=$ no change in position, and n.t. $=$ no test was performed

Sheep heads $1-4$ were used for the first tests of the GPII bullet, and sheep heads 5-8 were used for the first tests of the PP41 bullet; after the first series of tests, the brain tissues of the heads had not yet been lacerated due to previous insertions and tests of the other type of bullet. In the first test series, the GP11 bullet underwent migration once (in sheep head 7, after previous tests with the PP41 bullet); similarly, the PP41 bullet underwent migration only once (in sheep head 6). Across all of the movement tests over several days, bullet migrations occurred only occasionally ( $18.75 \%$ of tests), whereas bullet rotation was observed four times more often ( $75 \%$ of tests). Half of the observed migrations occurred in a single head (sheep head 2), in which migrations were consistently observed after the PP4I bullet first migrated in the second test series

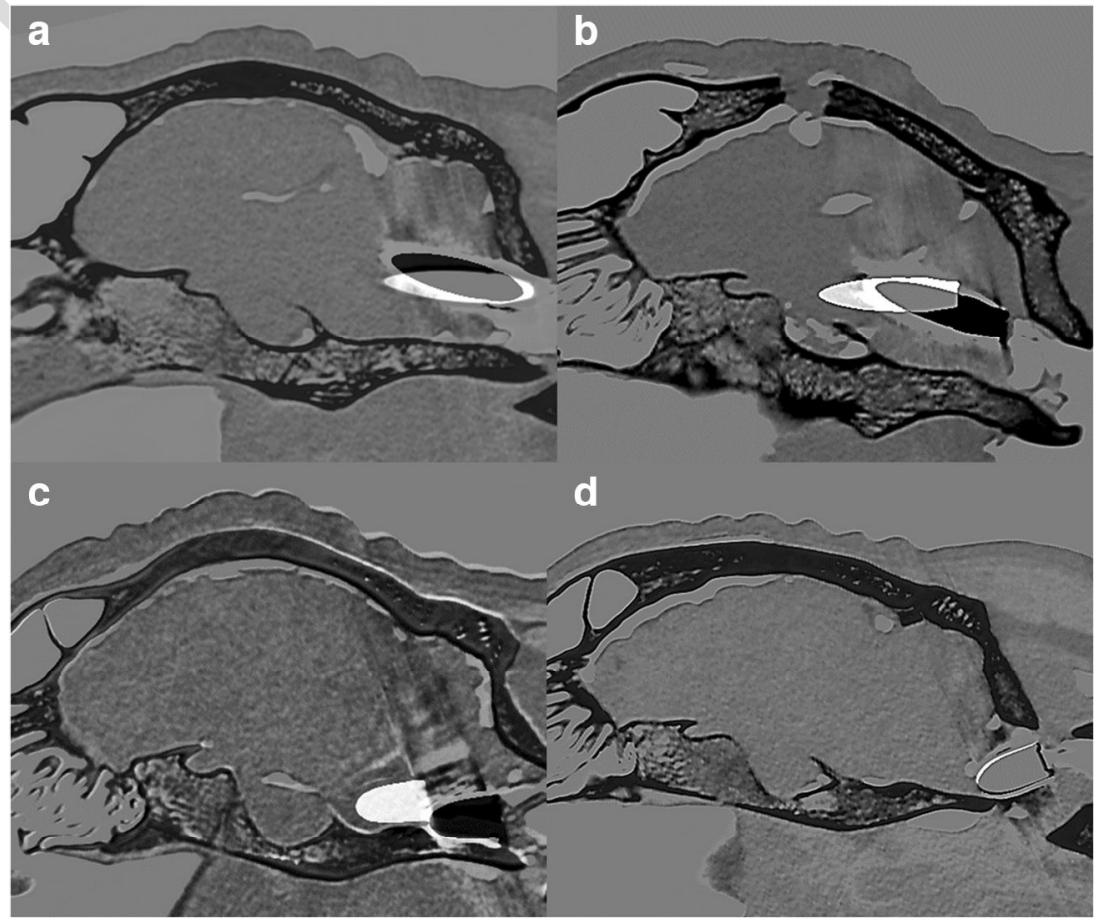




\section{Conclusions}

\section{Key points} animals in science. disclose.
Postmortem MRI is viable in cases of penetrating gunshot wounds with intact, deformed or fragmented bullets or pellets lodged inside the head. Postmortem MRI provides a valuable supplement to postmortem CT concerning the detection of wound channel and soft tissue injuries. MRI examinations may even provide further knowledge about wound ballistics since this imaging modality enables noninvasive precise visualization of soft tissue injuries in situ.

1. Postmortem MRI is a valuable supplement to postmortem $\mathrm{CT}$ in cases of gunshot wounds with regard to the detection of the wound channel and soft tissue lesions.

2. In the case of a transversal trajectory, the metal artifacts of retained bullets severely impede the identification of relevant findings on $\mathrm{CT}$, whereas non-ferromagnetic bullets and pellets cause only small artifacts on MRI.

3. Bullets do not present relevant temperature increases during a standard MRI examination of the head.

4. The likelihood of migration of ferromagnetic projectiles lodged inside brain tissue is low in cadavers with a short postmortem interval $(<12 \mathrm{~h})$.

5. Ferromagnetic projectiles align their longitudinal axis to the z-axis of the MRI scanner.

Acknowledgments The authors would like to thank their colleagues from the forensic institutes for providing information on the bullets. The authors would also like to thank Patrick Kircher from the Vetsuisse Faculty (University of Zurich) for endorsing the collaboration of these institutes in this field of research. The authors express their gratitude to Emma Louise Kessler for her donation to the Zurich Institute of Forensic Medicine, University of Zurich, Switzerland.

Funding This scientific paper received no external funding.

\section{Compliance with ethical standards}

This study was performed with human cadavers. Ethical approval was waived by the responsible ethics committee of the Canton of Zurich (waiver number: 2015-0686). This article does not contain any studies with (living) human participants. No animals were killed for the scientific purpose of this study. The animal models used in this study were obtained from a veterinary pathology institute. The fresh cadavers were subsequently used in other studies, according to the 3Rs (Replacement, Reduction, and Refinement) - the guiding principles for the more ethical use of

Conflict of interest The authors have no financial conflicts of interest to

\section{References}

1. Levy AD, Abbott RM, Mallak CT, Getz JM, Harcke HT, Champion HR, et al. Virtual autopsy: preliminary experience in high-velocity gunshot wound victims. Radiology. 2006;240:522-8.

2. Andenmatten MA, Thali MJ, Kneubuehl BP, Oesterhelweg L, Ross $\mathrm{S}$, Spendlove D, et al. Gunshot injuries detected by post-mortem multislice computed tomography (MSCT): a feasibility study. Legal Med. 2008;10:287-92.

3. Garetier M, Deloire L, Dédouit F, Dumousset E, Saccardy C, Ben SD. Postmortem computed tomography findings in suicide victims. Diagn Interv Imaging. 2017;98:101-12.

4. Thali MJ, Yen K, Vock P, Ozdoba C, Kneubuehl BP, Sonnenschein $\mathrm{M}$, et al. Image-guided virtual autopsy findings of gunshot victims performed with multi-slice computed tomography (MSCT) and magnetic resonance imaging (MRI) and subsequent correlation between radiology and autopsy findings. Forensic Sci Int. 2003;138: 8-16.

5. Stuehmer C, Blum KS, Kokemueller H, Tavassol F, Bormann KH, Gellrich NC, et al. Influence of different types of guns, projectiles, and propellants on patterns of injury to the viscerocranium. J Oral Maxillofac Surg. 2009;67:775-81.

6. Yong YE. A systematic review on ricochet gunshot injuries. Legal Med. 2017;26:45-51. Franckenberg S, et al. A moot point! A homicide case report on ambiguous projectile movement on postmortem MR. J Forensic Radiol Imaging. 2016;5:62-7.

8. Cha JG, Kim DH, Kim DH, Paik SH, Park JS, Park SJ, et al. Utility of postmortem autopsy via whole-body imaging: initial observations comparing MDCT and 3.0T MRI findings with autopsy findings. Korean J Radiol. 2010;11:395-406.

9. Jackowski C, Warntjes MJB, Kihlberg J, Berge J, Thali MJ, Persson A. Quantitative MRI in isotropic spatial resolution for forensic soft tissue documentation. Why and how? J Forensic Sci. 2011;56:20815.

10. Tschui J, Jackowski C, Schwendener N, Schyma C, Zech WD. Post-mortem CT and MR brain imaging of putrefied corpses. Int J Legal Med. 2016;130:1061-8.

11. Gascho D, Heimer J, Tappero C, Schaerli S. Relevant findings on postmortem CT and postmortem MRI in hanging, ligature strangu- 544 lation and manual strangulation and their additional value compared $\quad 545$ to autopsy - a systematic review. Forensic Sci Med Pathol. 546 2019;15:84-92

12. Smith AS, Hurst GC, Duerk JL, Diaz PJ. MR of ballistic materials: imaging artifacts and potential hazards. Am J Neuroradiol. 1991;12: $567-72$.

13. Hess U, Harms J, Schneider A, Schleef M, Ganter C, Hannig C. Assessment of gunshot bullet injuries with the use of magnetic resonance imaging. J Trauma. 2000;49:704-9.

14. Dedini RD, Karacozoff AM, Shellock FG, Xu D, McClellan RT, Pekmezci M. MRI issues for ballistic objects: information obtained at 1.5-, 3- and 7-tesla. Spine J. 2013;13:815-22.

15. Eggert S, Kubik-Huch RA, Klarhöfer M, Peters A, Bolliger SA, Thali MJ, et al. Fairly direct hit! Advances in imaging of shotgun projectiles in MRI. Eur Radiol. 2015;25:2745-53.

16. van Kan RAT, Haest IIH, Lahaye MJ, Hofman PAM. The diagnostic value of forensic imaging in fatal gunshot incidents: a review of literature. J Forensic Radiol Imaging. 2017;10:9-14.

17. Giorgetti A, Giraudo C, Viero A, Bisceglia M, Lupi A, Fais P, et al. Radiological investigation of gunshot wounds: a systematic review of published evidence. Int J Legal Med. 2019;133:1149-58.

18. Oehmichen M, Gehl HB, Meissner C, Petersen D, Höche W, Gerling I, et al. Forensic pathological aspects of postmortem 
imaging of gunshot injury to the head: documentation and biometric data. Acta Neuropathol (Berl). 2003;105:570-80.

19. Abdul Rashid SN, Martinez RM, Ampanozi G, Thali MJ, Bartsch C. A rare case of suicide by gunshot with nasal entry assessed by classical autopsy, post-mortem computed tomography (PMCT) and post-mortem magnetic resonance imaging (PMMR). J Forensic Radiol Imaging. 2013;1:63-7.

20. Achenbach S, Moshage W, Diem B, Bieberle T, Schibgilla V, Bachmann K. Effects of magnetic resonance imaging on cardiac pacemakers and electrodes. Am Heart J. 1997;134:467-73.

21. Konings MK, Bartels LW, Smits HFM, Bakker CJG. Heating around intravascular guidewires by resonating RF waves. J Magn Reson Imaging. 2000;12:79-85.

22. Saukko P, Knight B. Electrical fatalities. In: Saukko P, Knight B, editors. Knight's forensic pathology. 4th ed. Boca Raton: CRC Press; 2016. p. 325-38.

23. Guo R, Chen M, Zheng J, Yang R, Chen J, Kainz W. Comparison of in-vivo and in-vitro MRI RF heating for orthopedic implant at 3 tesla. 2017 IEEE Int Symp Electromagn Compat Signal Power Integr EMCSI. 2017. pp. 123-8.

24. Yeung CJ, Susil RC, Atalar E. RF safety of wires in interventional MRI: using a safety index. Magn Reson Med. 2002;47:187-93.

25. Luechinger R, Zeijlemaker VA, Pedersen EM, Mortensen P, Falk E, Duru $F$, et al. In vivo heating of pacemaker leads during magnetic resonance imaging. Eur Heart J. 2005;26:376-83.

26. Bolliger SA, Thali MJ, Gascho D, Poschmann SA, Eggert S. Movement of steel-jacketed projectiles in biological tissue in the magnetic field of a 3-T magnetic resonance unit. Int J Legal Med. 2017;131:1363-8.

27. Diallo I, Auffret M, Attar L, Bouvard E, Rousset J, Salem DB. Magnetic field interactions of military and law enforcement bullets at 1.5 and 3 tesla. Mil Med. 2016;181:710-3.

28. Eggert S, Kubik-Huch RA, Lory M, Froehlich JM, Gascho D, Thali $\mathrm{MJ}$, et al. The influence of 1.5 and $3 \mathrm{~T}$ magnetic resonance unit magnetic fields on the movement of steel-jacketed projectiles in ordnance gelatin. Forensic Sci Med Pathol. 2015;11:544-51.

29. Luijten M, Haest IIH, van Kan RAT, van Lohuizen W, Kroll J, Schnerr RS, et al. Can postmortem MRI be used to assess trajectories in gunshot victims? Int J Legal Med. 2016;130:457-62.

30. Gascho D, Philipp H, Flach PM, Thali MJ, Kottner S. Standardized medical image registration for radiological identification of decedents based on paranasal sinuses. J Forensic Legal Med. 2018;54: 96-101.

31. Gascho D, Flach PM, Schaerli S, Thali MJ, Kottner S. Application of 3D image fusion for radiological identification of decedents. $\mathrm{J}$ Forensic Radiol Imaging. 2018;13:12-6.

32. Hartwig V, Giovannetti G, Vanello N, Lombardi M, Landini L, Simi S. Biological effects and safety in magnetic resonance imaging: a review. Int J Environ Res Public Health. 2009;6:1778-98.

33. Gascho D, Thali MJ, Niemann T. Post-mortem computed tomography: technical principles and recommended parameter settings for high-resolution imaging. Med Sci Law. 2018;58:70-82.

34. Berger F, Niemann T, Kubik-Huch RA, Richter H, Thali MJ, Gascho D. Retained bullets in the head on computed tomography - get the most out of iterative metal artifact reduction. Eur J Radiol. 2018;103:124-30.

35. Riva F, Lombardo P, Zech WD, Jackowski C, Schyma C. Individual synthetic head models in wound ballistics - a feasibility study based on real cases. Forensic Sci Int. 2019;294:150-9.

36. Gupte AA, Shrivastava D, Spaniol MA, Abosch A. MRI-related heating near deep brain stimulation electrodes: more data are needed. Stereotact Funct Neurosurg. 2011;89:131-40.

37. Kainz W. MR heating tests of MR critical implants. J Magn Reson Imaging. 2007;26:450-1.

Publisher's note Springer Nature remains neutral with regard to jurisdictional claims in published maps and institutional affiliations. 